\title{
EVOLUTION OF PN MORPHOLOGIES: CONCEPTS, MODELS AND OBSERVATIONS
}

\author{
BRUCE BALICK \\ Astronomy Department, FM-20, University of Washington, Seattle WA 98195, U.S.A.
}

\begin{abstract}
The shapes of planetary nebulae (PNs) provide paleontological clues about the origin and evolution of the gas expelled in the late phases of stellar evolution. The morphological classes of planetaries and various structural components of the nebulae are interpreted as hydrodynamic interactions of episodes of relatively brief, axisymmetric winddriven mass-loss events. Theoretical studies of the past five years are compared with extant data to show that astrophysical hydro models are achieving a very high level of success as explanations for the shapes of most PNs.

The most successful models are those for which the star is assumed to expel much or most of its mass in an equatorial wasteband. In stark contrast are dense ansae, dense and lowionization knots of fast-moving gas, for which the mass distribution is decidedly polar. The origins of both the equatorial wastebands and polar knots remain decidedly enigmatic.
\end{abstract}

\section{Introduction}

PN morphologies have been classified in many different ways. Recent classification schemes seem to have in common that PN shapes span the extrema of circular nebulae (containing various concentric components shown schematically in fig. 1) and highly bipolar structures

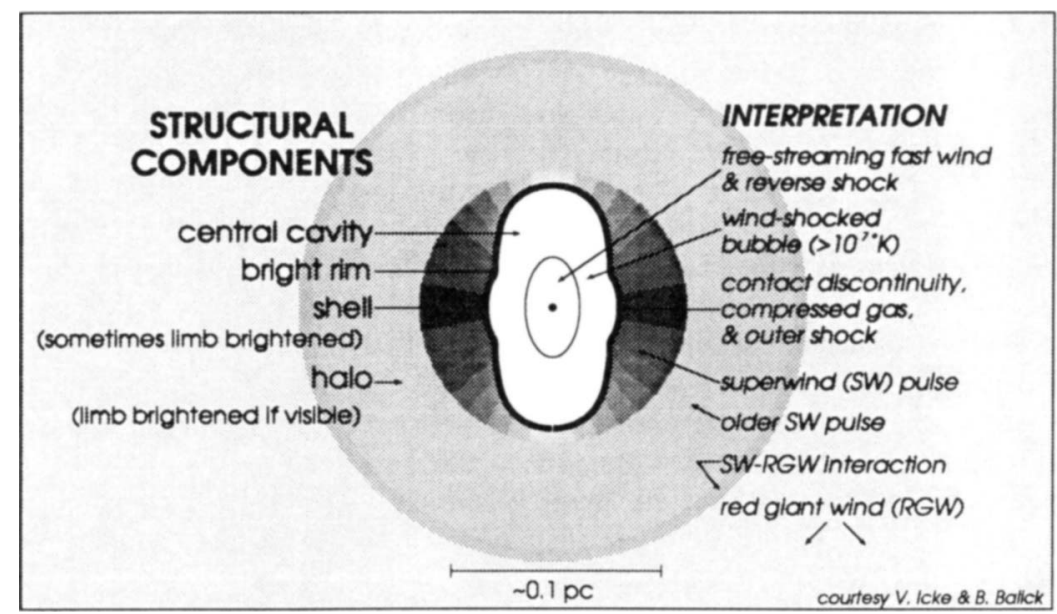

Fig. 1. Major PN components and their hydrodynamic interpretation

R. Weinberger and A. Acker (eds.), Planetary Nebulae, 131-138.

(C) 1993 IAU. Printed in the Netherlands. 
with prolate elliptical structures of various types in between (e.g. Balick [1]; Schwarz et al. ${ }^{*}$ and Romano et al. ${ }^{*}$ ). The PNs in each morphological class have highly characteristic properties. There is strong evidence that much of the axisymmetry is imposed in the protoPN phase (e.g. Sahai, et al. ${ }^{*}$, Bachiller et al. ${ }^{*}$, Shibata et al. ${ }^{*}$, Wolstencroft et al. $\left.{ }^{*},[2]\right)$.

The organized shapes of PNs and their structural components contain information about the history of the nebula, especially the ways in which material is ejected from the rapidly evolving nucleus. These clues can be read if the processes that govern the evolution of the expelled gas can be understood.

The idea that PNs eject mass in episodes, each marked by different mass loss rates, velocities, and ejection patterns, has become soundly established in the past five years. Kwok, Purton, and their many collaborators introduced and established the concept of interacting winds into our field a decade ago. Their calculations assumed spherical symmetry.

Balick [1] argued that only a tiny fraction of PNs appear circular; most are far more interesting and needed to be investigated theoretically using two dimensional hydro. The seminal work in the realm of two-dimensional mass loss was that of Kahn and West [3], who assumed that early episodes of mass ejection expelled mass preferentially in an equatorial plane. They showed that a fast, symmetric wind ejected later that interacts with the older ejecta can produce elliptical or peanut nebular symmetry. Various groups (e.g. Soker and Livio; Balick, Icke, Frank, and Mellema [4-10] and Icke* ${ }^{*}$ Mellema*, Igumenschev*, Zweigle et al. ${ }^{*}$, Pascoli", Diesch and Grewing ${ }^{*}$, Sahu et al. ${ }^{*}$, and Frank's string talk) expanded upon this idea in some detail through extensive observations of kinematics and the development of both analytical and numerical hydrodynamic models containing many of the important physical processes that were not treated earlier. The highlights of these papers are reviewed here, and details can be found in specific papers cited later.

Because of their brightness and ease of observations, PNs are serving as a crucial testing ground of astrophysical hydrodynamics [8]. PNs have served physics faithfully in the same mode for theories of atomic structure and ionization and cooling processes.

\section{Morphological Classes}

We shall adopt Balick's qualitative two-parameter morphology classes here. One parameter is the degree of departure from roundness which varies continuously from round to elliptical to bipolar. The other is overall physical size, taken to be an indication of age, and so designated early, middle, and late. Round nebulae remain round as they evolve, but the degree of asymmetry increases in other classes as the fast stellar wind interacts with the structured slow wind. For example, nebulae which are peanut shaped in an early evolutionary state were assumed to develop into bipolars with open lobes in later stages. Balick's morphology classes were really simply guesses about the likely hydrodynamic evolution of nebulae which depend only on the "initial condition" of the equatorially enhanced mass distribution of the slow wind and the "boundary condition" of the speed and mass flux of the fast wind. (He assumed the nebulae to be isothermal, an assumption that is challenged by newer observational and theoretical studies.)

The hydro models presented here are broadly consistent Balick's classification, although the model predictions to be described here show a richer variety of morphologies. A review of the computations is the focus of this talk.

* This symbol denotes a contribution to the present conference. 


\section{Structural Components}

\subsection{HALOS}

Halos are the largest and (by assumption) the oldest ejected material still visible. Halos are reviewed by Meaburn, Dyson, Frank, Balick, Chu and their many collaborators [12-17]; see also Kwitter et al. ${ }^{*}$, Lopez et al. ${ }^{*}$. Halo masses are comparable to those of the interior structure $\left(\approx 0.25 \mathrm{M}_{\odot}\right)$ [15]. Frank et al. [16] have argue that halos are characterized by ages of more than $10^{4} \mathrm{y}$. Frank and Balick ${ }^{*}$ propose that they arise from episodic superwind ejections associated with thermal pulses of the central star.

Morphologically, halos are larger, fainter, and rounder than the structure interior to them [17] and are always limb brightened, suggesting significant ram pressure by an external confining medium. Some PNs, e.g. A 16, 30, 33, 34, and 39, appear to be halo-dominated with little or no interior structure. (A few halos are distorted by their motion relative to the local ISM [17-19].) No halo has been found to be associated with a bipolar PN, though this may be an artifact of ionization shadowing and the exact definition of "bipolar PN". Most halos expand at or below the sound speed (Bryce et al. ${ }^{*},[12-14]$ ) - the unusual halo of NGC 7662 is a likely exception [12] - and have slightly higher excitation temperatures [15].

The roundness of halos may indicate that early superwind ejections are less structured than later pulses. Alternately the roundness can evolve from hydrodynamic effects. Halos might eventually form as expanding bipolar lobes merge into one large bubble; NGC 2371-2 and 2440 may be examples.

As yet there are no detailed models of the growth of haloes. Proper calculations are difficult; the form, duration, and axisymmetries of the mass distribution of a superwind pulse (Vassiliadis and Wood", [20]); the gas "softening" effects of uv photon heating; and radiative cooling mechanisms for neutral gas must all be included.

\subsection{SHELLS}

Shells are smaller, brighter, (presumably) younger than halos, and universally attached to interior bright rims. Occasionally they are also limb brightened, hinting at the presence of a partially confining medium (e.g. a halo). Frank et al. [16] showed that between the interior rims and exterior limbs shell emission measures (EM) decline linearly, and not $\mathrm{r}^{-3}$ as might be expected from a steady wind. Shells show enhanced EM along their narrowest axis which is always orthogonal to the longest symmetry axis of the nebula, as illustrated in fig. 2. (See Bremer and Grewing ${ }^{*}$, Volk and Leahy ${ }^{*}$, and Soker et al. [21] for mapping techniques.)

Kahn, Balick and many others [1,3-11] assert that regions of enhanced EM arise from the higher-density, equatorial superwind wasteband. This forces PNs to grow preferentially along their poles. More precisely, an invisible fast wind blowing from the central star, characterized by a speed of $10^{3} \mathrm{~km} \mathrm{~s}^{-1}$ and a mass loss rate $\approx 10^{-7} \mathrm{M}_{\bullet}$, presently interacts with the shell (i.e. the latest pulse of superwind material) forming a strong shock and, with it, a region of snowplowed material which forms the bright rim inside the shell. Detailed hydrodynamic models of the evolution of such a system are form the Ph.D. theses of A. Frank and G. Mellema; encouraging recent results are being presented here (see Frank ${ }^{*}$, Mellema*, and the evolving hydro model video by Icke $^{*}$ ), and others appear in the literature [4-11]. 


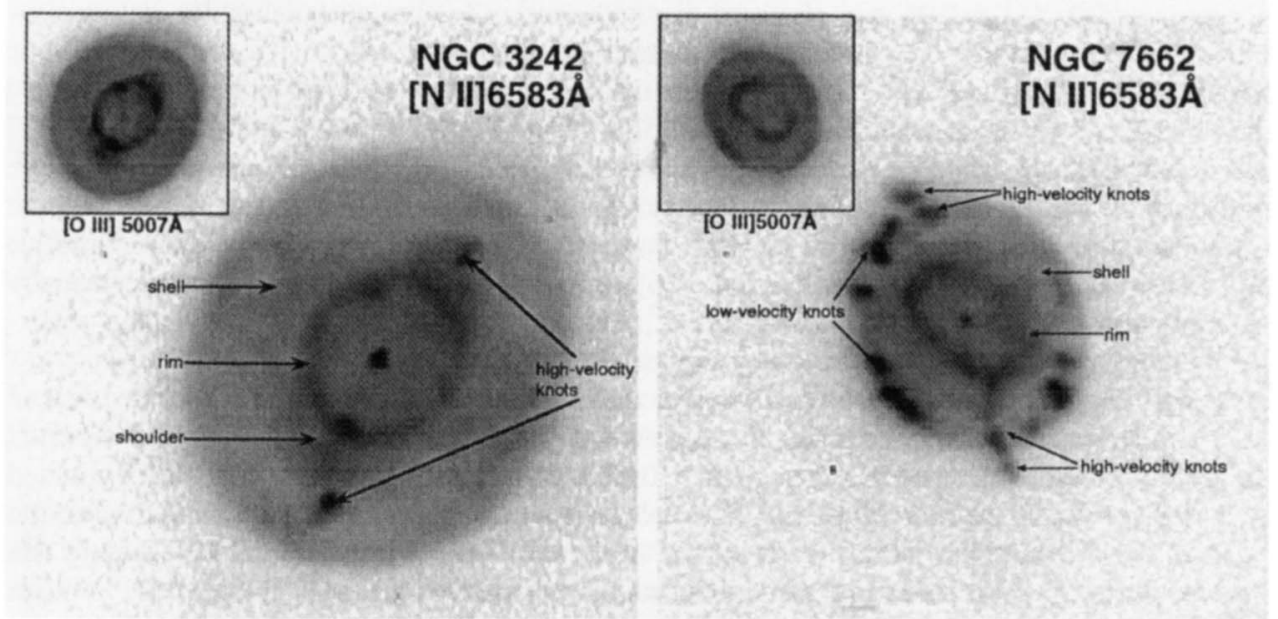

Fig. 2. Shells and Other Features of Elliptical PNs

Generally speaking, IR $\mathrm{H}_{2}$ lines are distributed at the outer edges of shells where [O I] and [N II] lines are bright. For NGC 6720 and 6853 in which $\mathrm{H}_{2}$ has been mapped, the molecules arise inside the ionized halo at the outer perimeter of the shell [e.g., 17].

\subsection{CORES AND RIMS}

Cores (i.e. central cavities) and the bright rims which surround them and define their geometry are ubiquitous amongst almost all PNs. The rims are generally described as having round, elliptical, peanut, and butterfly (or bipolar) shapes.

The interacting wind model accounts nicely for the morphological features of rims. A fast stellar wind enters and shocks material in the cavity to $10^{7-8} \mathrm{~K}$, creating a hot bubble at higher pressure than its surroundings (i.e. the latest pulse of superwind ejecta). The bubble expands supersonically into the denser, cooler gas outside of it $\left(\mathrm{T} \approx 10^{4}{ }^{\circ} \mathrm{K} ; \mathrm{n} \approx 10^{3} \mathrm{~cm}^{-3}\right)$. In the process the interface region is shocked and compressed nearly isothermally, owing to the highly effective cooling processes available to the cooler gas. The compressed gas appears in projection as the rims. The hot bubble is invisible at optical-uv wavelengths.

The shape of the rims is governed by the initial distribution of the superwind ejecta which forms the shell. Since shells tend to have toroidal distributions, the hot bubble grows much faster towards the toroid's poles. Once the elliptical bubble "breaks out" along the poles the nebular shape becomes bipolar. Expansion velocities can be huge since the poorly confined hot gas tends to expand along the poles at its own sound speed of $\geq 50 \mathrm{~km} \mathrm{~s}^{-1}$.

A snapshot of the early evolution of the hot bubble and its rim-like boundary is illustrated in fig. 3 under two sets of initial conditions. The models are from Mr. Frank's $\mathrm{Ph} . \mathrm{D}$. thesis and will be described by him in more detail in this conference. See also Icke $e t$ al. [8] for detailed models which neglect energy loss by radiation; such models could be applicable during the protoPN phase if radiative cooling is unimportant. Similar results were obtained by Soker and Soker and Livio $[9,11]$ using a radiationless and numerically less precise "particle-in-cell" methodology. 


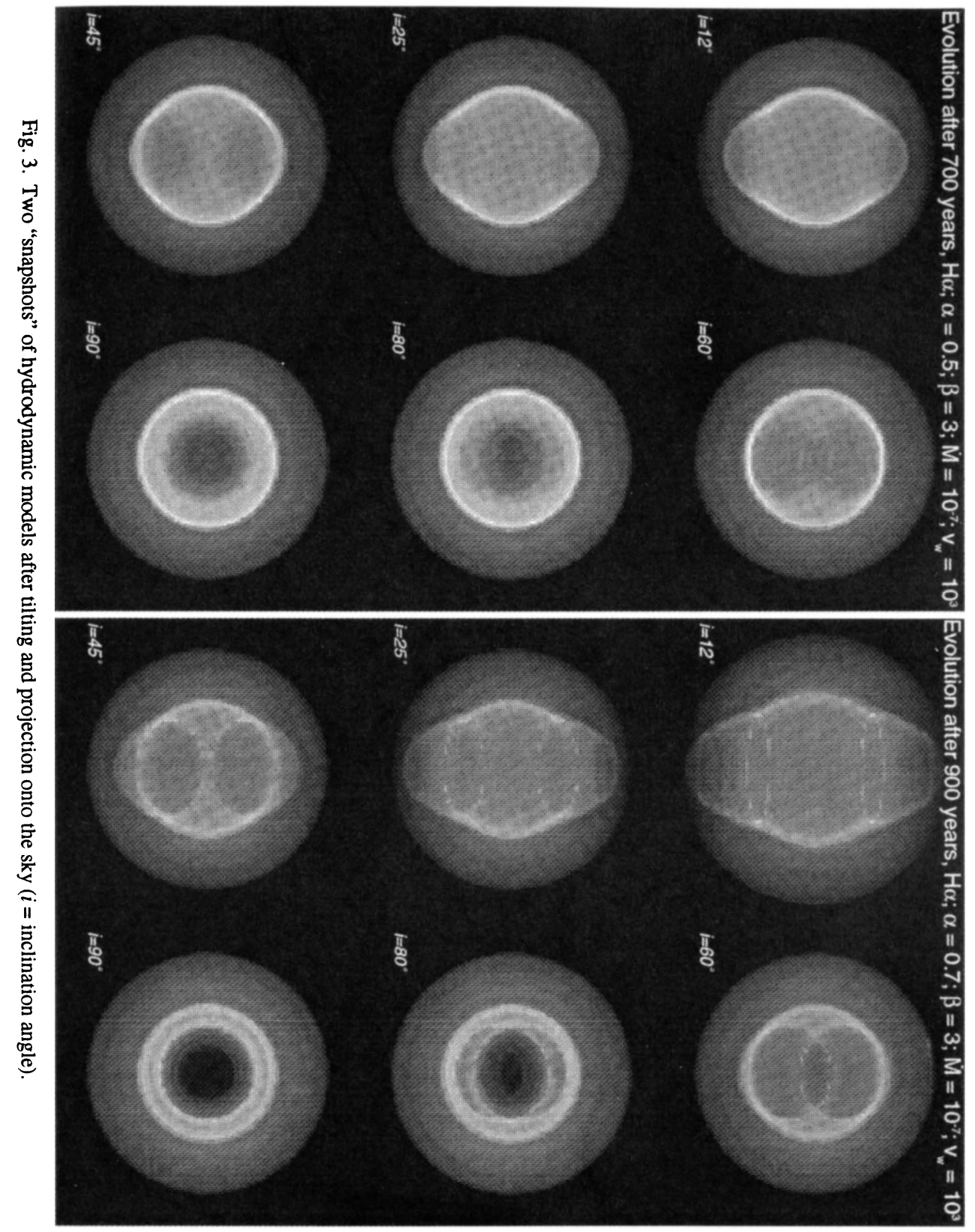




\section{Kinematics}

Kinematics are, in a sense, the first derivative of the structure; they show how the structure evolves into the next era. More than this, kinematics are crucial tests of models. Model builders twiddle their parameters until they fit the morphologies. Until the models successfully predict the kinematics, they are little more than academic homework problems.

Space does not permit an adequate discussion of nebular kinematics. Over the past five years many observations at $\geq 10 \mathrm{~km} \mathrm{~s}^{-1}$ spectral resolution have appeared. Balick, Icke, O'Dell, and their many collaborators have mapped the two-dimensional kinematics of various PNs in $\mathrm{H} \alpha,[\mathrm{N} \mathrm{II}]$, and [O III] lines. See also Pismis et al. ${ }^{*}$, Diesch and Grewing*, and Sahu et al.*.

PNs have complex motions not well characterized by a single expansion velocity. NGC 2392, studied in greatest detail by O'Dell et al. [22] is an excellent case in point. The brightest inner nebulosity has velocities which span about $80 \mathrm{~km} \mathrm{~s}^{-1}$. Outer, fainter nebulosity in the nebula's shell spans twice this range of velocities, and two small features appear at $\pm 180 \mathrm{~km} \mathrm{~s}^{-1}$ on opposite sides of the star. Most other nonbipolar PNs exhibit velocities which decrease at larger radii from the nucleus. As for the bipolars, the most detailed analysis is by Icke et al. [7] who find considerable nebula-to-nebula differences.

The interpretation of the kinematics is rich in ideas. Hydro models which are careful to permit conversion of kinetic (and thermal) energy into radiation are just now appearing for the first time. So far the results are sketchy. Nonetheless it is clear from one-dimensional calculations (some of which are to be presented by Frank) that the patterns of kinematics change qualitatively as shocks and ionization fronts propagate beyond various structural components of PNs. Generally the hot bubble expands into the shells and, so, accounts for a negative velocity gradient with radius. However, there are times when shocks propagate through the confining medium, and the velocity gradient tends towards zero.

Two-dimensional models (e.g. Frank*, Icke*, and Mellema*) show complex patterns of motions which are generally consistent with the various various data mentioned above.

\section{Puzzles}

Most all of the large morphological features of PNs. such as rims, shells, and halos, are qualitatively understandable if a theoretician is free to pick initial and boundary conditions for the models. Our ignorance hides in these choices: in what patterns do stars eject their mass prior to the PN stage of evolution (and why??); what are the time-dependent spectral and wind characteristics of the nucleus (and why??); etc.

The mechanisms of mass ejection are only poorly understood, and it is truly a puzzle how a star can eject material with quadrapole and higher moments in its distribution. Rotation, chaotic or nonradial pulsations, magnetic fields intermal and external to the star, contact or close binaries; all of these mechanisms and others are all being explored. It is safe to say that no consensus is emerging, and that detailed images of protoPNs, such as the many results being reported here, are crucial to constrain our imaginations.

The wind characteristics of fast winds have been probed, primarily along the line of sight ending at the star. P-Cygni profiles have been observed in many ultraviolet lines and in many nuclei. Whether the winds are spherically symmetric, and how they evolve in time are open questions whose answers are likely to come eventually from theoretical models. 
On the smaller scale, low-ionization knots of various distributions are common in PNs, as illustrated in fig. 2. Often the knots appear along the outer perimeter of shells (e.g. the lowvelocity knots of NGC 7662 and 2392). Kahn and Breitschwerdt [23,24] argue that expansion of the PN in certain phases of its evolution can generate Rayleigh-Taylor instabilities and fragment the shell. They argue that the low-ionization knots are short-lived.

Pairs of knots along or near the nebular major axis, called "ansae", are not uncommon (fig. 2). The ansae are characterized by spectra very similar to Herbig-Haro objects in the strengths of their [S II], [O I], [N I], and $\mathrm{Mg} \mathrm{I}$ line intensities and the weakness of all permitted lines, including $\mathrm{H} \alpha$. These unusual line ratios suggest that the ansae are partially or completely collisionally excited by shocks with velocities of $50-80 \mathrm{~km} \mathrm{~s}^{-1}$. Almost certainly ansae are plowing outward through the nebula along a symmetry axis. Many ansae are embedded in gas of much higher ionization. Curiously enough, Echelle observations show exactly these characteristic velocities for the ansae after reasonable correction for projection effects!

Ansae are always associated with elliptical PNs except for IC 2149 in which the ansae are the only bright nebular features. Some PNs, such as NGC 2440 and 5189, show several pairs of ansae along axes with different P.A.s. Other PNs and protoPNs exhibit ansae only as molecular or dusty features (e.g. Morris [25], Sahai et al. .). Abell 30, a very old and large $\mathrm{PN}$, has $\mathrm{N}$-rich ansae as well. In other words, ansae are apparently ejected in symmetric pairs as early as the protoPN phase and throughout the subsequent lifetime of the PN. What are the ansae? How are they ejected? Only the central star knows for sure.

That $\mathrm{H}_{2}$ is even detectable is somewhat surprising given that many host PNs are not ionization bounded. Even more enigmatic is why the $\mathrm{H}_{2}$ in NGC 6720 and 6853 is found between their respective highly ionized halos and the interior shells.

Finally we note that although the existence of halos is no surprise, we have much to learn from them about the ways in which superwinds are ejected in the protoPN phase or even earlier (see reviews by $[21,26]$ ). Some halos develop comet-shaped knots, others are mottled, and yet others are smooth. Halos seem to provide fertile conditions in which to probe the formation of astrophysical instabilities, and discussed by Meaburn, Dyson, Bryce, and collaborators $[13,14]$.

\section{Summary}

What have we learned since the 1987 IAU Symposium on PNs in Mexico City about the morphologies and evolution of PNs? The crucial importance of continuing wind shaping is firmly established. Most PN features can be well understood in terms of natural hydrodynamic process. Two-dimensional hydrodynamic models are now climbing onto very solid ground as the tools for understanding all of the gross morphological and kinematic features, and perhaps the fine-scale structure $(\leq 0.01 \mathrm{pc})$ structure, of PNs. We understand that shocks are very important in the shaping and line excitations of PNs, particularly in the ansae. All of the morphological characteristics of PNs appear in protoPNs as well, so stellar mass loss and the attendant hydrodynamic processes that shape PNs exert their influence before some PNs become visible.

Yet we are sobered by the various puzzles listed in the previous section. And a detailed model is yet to be successfully computed for one very intensively studied PN, such as NGC 6751 [27], albeit with unlimited freedom to choose initial and boundary conditions! 


\section{Acknowledgements}

It is a sincere pleasure to thank my close colleagues and friends, V. Icke, A. Frank, and G. Mellema, for their collaborations, driving enthusiasm, and warm hospitality. N. Soker, F. Kahn, and J. Dyson have engaged me in many insightful and thought-provoking conversations. Many other colleagues throughout the world who make the work reported here so stimulating and enjoyable.

Above all, I would like to thank the many authors of the 37 poster papers that are directly relevant to this talk, and to apologize to everyone whose work I could not or did not reference explicitly herein. These fabulous poster papers clearly define the state of the theoretical and observational art of our science at this time.

\section{References}

1 Balick, B. (1987) Astron. J. 94, 671

2 Morris, M. and Reipurth, B. (1990) Publ. Astron. Soc. Pacific 102, 446

3 Kahn, F. and West, K.A. (1985) Mon. Not. Roy. Astron. Soc. 212, 837

4 Balick, B. Preston, H.L., and Icke V. (1987) Astron. J. 94, 1641

5 Icke, V. (1991) Astron. Astrophys. 251, 369

6 Icke, V., Balick, B., and Frank, A. (1992) Astron. Astrophys. 253, 224

7 Icke, V., Preston, H.L., and Balick, B. (1989) Astron. J. 97, 462

8 Icke, V., Mellema, G., Balick, B., Eulderink, F., and Frank, A. (1992) Nature, 355, 524

9 Soker, N. (1989) Astrophys. J. 340, 921

10 Soker, N. (1990) Astron. J. 99, 1869

11 Soker, N. and Livio, M. (1989) Astrophys. J. 339, 268

12 Chu, Y.-H. and Jacoby, G.H. (1989) in IAU Symp. 131, Planetary Nebulae, S. Torres-Peimbert (ed.) Kluwer Academic Publishers, Dordrecht, p. 198

13 Meaburn, J., Nicholson, R. Bryce, M., Dyson, J.E., and Walsh, J.R. (1992) Mon. Not. Roy. Astron. Soc. 252, 535

14 Bruce, M., Meaburn, J., Walsh, J.R., and Clegg, R.A.S. (1992) Mon. Not. Roy. Astron. Soc. 254, 477

15 Middlemass, D., Clegg, R.A.S., Walsh, J.R., and Harrington, J.P. (1991) Mon. Not. Roy. Astron. Soc. 251, 284

16 Frank, A., Balick, B., and Riley, 1990, Astron. J. 100, 1903

17 Balick, B., Gonzalez, G., Frank, A., Jacoby, G. (1992) Astrophys. J. 392, 582

18 Borkowski, K.J., Sarazin, C.L., and Soker, N. (1990) Astrophys. J. 360, 173

19 Soker, N., Borkowski, K.J., and Sarazin, C.L. (1991) Astron. J. 102, 381

20 Wood, P.R. and Vassidiadis, E. (1992) Late Evolution of Low-Mass Stars, (to appear in Highlights of Astronomy, vol.. 9) Kluwer Academic Publishers, Dordrecht

21 Soker, N., Zucker, D.B., and Balick, B. (1992) subm. to Astrophys. J.

22 O'Dell, R., Weiner, L.D., and Chu, Y.-H. (1990) Astrophys. J. 362, 226

23 Breitschwerdt, D. and Kahn, F.D. (1990) Mon. Not. Roy. Astron. Soc. 244, 521

24 Kahn, F.D. and Breitschwerdt, D. (1990) Mon. Not. Roy. Astron. Soc. 242, 505

25 Morris, M. (1987) Publ. Astron. Soc. Pacific 99, 1115

26 Pascoli, G. (1992) Publ. Astron. Soc. Pacific 104, 350

27 Chu, Y.-H., Manchado, A., Jacoby, G.H., and Kwitter, K.B. (1991) Astrophys. J. 376, 150 